\title{
Cardiac nodal and cardiac autonomic functions in children with vasovagal syncope
}

\author{
Serhat Koca ${ }^{1}$, Feyza A. Paç ${ }^{1}$ İbrahim Ece ${ }^{1}$, Denizhan Bağrul ${ }^{1}$, Serkan Çay ${ }^{2}$ \\ ${ }^{1}$ Department of Pediatric Cardiology and ${ }^{2}$ Division of Arrhythmia and Electrophysiology, Department of Cardiology, \\ Yuksek Ihtisas Heart -Training and Research Hospital, Ankara, Turkey. \\ E-mail: drserhatkoca@gmail.com \\ Received: 24th May 2016, Revised: 23rd January 2017, Accepted: 21st February 2017
}

\begin{abstract}
SUMMARY: Koca S, Paç FA, Ece I, Bağrul D, Çay S. Cardiac nodal and cardiac autonomic functions in children with vasovagal syncope. Turk J Pediatr 2016;
\end{abstract} 58: 498-502.

In the present study, cardiac nodal (sinoatrial node and atrioventricular node) and cardiac autonomic functions have been investigated in children with vasovagal syncope (VVS). Thus, the effect of existing autonomic status on the characteristics of cardiac conduction system has been demonstrated in children with VVS. The study included 51 pediatric patients (the mean age was $14.01 \pm 2.79$ years, range 7 to 18 years; 30 females), who were evaluated for syncope using ECG, Holter monitoring, and echocardiography, all of which showed normal findings. All patients underwent head-up tilt testing (HUTT), and the parameters of heart rate variability (HRV) (SDNN, SDANN, SDANNi, rMSSD, pNN50, HF, LF, VLF) were evaluated with the analysis of 24-hour Holter testing. Corrected sinus node recovery time (CNRT) and Wenckebach point (WP) were measured in all patients using transesophageal atrial stimulation. The patients were divided into groups according to HUTT results and evaluated accordingly. Cardiac nodal functions were normal in all patients. HRV parameters (HF, rMSSD, pNN50) indicating parasympathetic effect were higher in patients with positive HUTT results. CNRT did not differ significantly between patients with positive HUTT results and patients with negative HUTT results. However, WP was found to be higher in patients with positive HUTT results. The studies evaluating the parameters of HRV has demonstrated increased parasympathetic tonus in children with VVS. Impairment in cardiac nodal functions may not be expected in children with VVS. However, these patients may exhibit prolonged WP due to increased autonomic tonus in favor of parasympathetic activity.

Key words: vasovagal syncope, heart rate variability, sinoatrial node, atrioventricular node.

Syncope is a transient loss of consciousness characterized by the loss of posture and tonus and spontaneous recovery. Global cerebral hypoperfusion triggered by various factors leads to syncope ${ }^{1}$. Syncope occurs in $15 \%$ of children before adolescence. The most frequent cause of syncope in children is the vasovagal syncope (VVS) 2,3. The underlying mechanism in vasovagal syncope is the imbalanced autonomic response to orthostatic stress ${ }^{4}$. The analysis of heart rate variability using 24-hour Holter monitoring is a widely accepted method in the evaluation of autonomic nervous system ${ }^{5}$. There are studies evaluating sympathovagal balance in children suffering from syncope by investigating the parameters of heart rate variability (HRV). However, these studies have yielded controversial results ${ }^{6-10}$.

Dysfunction of sinoatrial node and atrioventricular node is another rare cause of syncope ${ }^{3}$. These clinical conditions can be determined by the evaluation of cardiac nodal functions with transesophageal electrophysiologic studies (TEEPS) ${ }^{11,12}$. There is no study that evaluated cardiac nodal function in pediatric cases suffering from vasovagal syncope. We have considered that autonomic state in children with vasovagal syncope 
might affect the characteristics of cardiac nodal tissue. In the present study, we aimed to evaluate the cardiac nodal (sinoatrial and atrioventricular nodes) functions, and the autonomic characteristics of heart in children with vasovagal syncope.

\section{Material and Methods}

A total of 51 patients with a history of vasovagal syncope aged between 7 and 18 years (mean age $14.01 \pm 2.79$ years; 30 females) were recruited between January 2012 and November 2015. Autonomic symptoms were present in all patients which was in favor of vasovagal syncope. Also, there was no history of exercise induced syncope or seizure in these patients which would make vasovagal syncope unlikely. None of the patients had apparent heart disease. Neurological evaluation of these patients made by pediatric neurologist were also normal. There was no familial history of unexplained sudden death or epilepsy. Clinical examination, surface ECG and bi-dimensional echocardiogram were normal in all patients. The patients with pre-excitation syndrome and conduction disturbances on ECG were excluded from study. In all patients, twentyfour-hour ECG Holter monitoring showed negative findings. Exercise stress test was performed in all patients and there were no ventricular tachycardia or ST-T changes in the patients. Syncope was thought secondary to be non-cardiac reason in patients with no family history of sudden cardiac death, normal physical exam, normal ECG, echocardiography, $24 \mathrm{~h}$ Holter and exercise stress test results.

Head-up tilt test (HUTT): All patients underwent standard HUTT using the Westminster protocol: passive phase of 60-degree tilt for 45 minutes. Drugs that could influence normal autonomic nervous system activity were stopped at least 3 days before the test. Heart rate, blood pressure and ECG were recorded until a positive response appeared or until the conclusion of the 45-minute test. The test was considered positive if the patient developed presyncope or syncope associated with a sudden fall in systolic blood pressure (of $>40 \mathrm{mmHg}$ ) and/ or a sudden drop in the heart rate (to $<50$ beats/minute) or if sinus rhythm was replaced by a junctional escape rhythm or if a transient second degree atrioventricular block or up to
3 seconds of asystole appeared. Negative tests were defined as the absence of these findings after the test. Based on the results of HUTT, the patients were divided into two groups; positive response (Group 1) and negative response (Group 2).

Holter monitoring and heart rate variability: The following parameters of the recorded ECG were evaluated: minimal rate of the heart contractions (bpm) recorded during $24 \mathrm{~h}$ (HR $\mathrm{min})$, mean rate of the heart contractions (bpm) recorded during $24 \mathrm{~h}$ (HR mean), and maximal rate of the heart contractions (bpm) recorded during $24 \mathrm{~h} \mathrm{HR}$ maximum (HR max). Twentyfour hour Holter recordings were obtained using 12-channel analogue recorders using the Cardioscan Holter system (Cardioscan DM Software, California, USA). The time-domain HRV measures employed in our practice were as follows:

SDNN (ms): standard deviation values of all normal sinus R-R intervals over $24 \mathrm{~h}$;

SDANN (ms): standard deviation values of all averaged normal sinus $\mathrm{R}-\mathrm{R}$ intervals for each 5 -min segment in the 24-h recordings;

SDANNi (ms): standard deviation of all normal $\mathrm{R}-\mathrm{R}$ intervals for all 5-min segments

rMSSD (ms): root mean squares of successive differences between normal sinus R-R intervals;

pNN50 (\%): percentage of difference between adjacent normal $\mathrm{R}-\mathrm{R}$ intervals that is greater than $50 \mathrm{~ms}$ computed over the entire 24-h ECG recording.

Spectral analysis of HRV was obtained by summing powers of each frequency band: high-frequency (HF) component (0.15-0.40 $\mathrm{Hz}$ ); low-frequency (LF) component (0.04-0.15 $\mathrm{Hz}$ ); and very-low-frequency (VLF) component (0-0.04 Hz).

Only normal QRS complexes were analyzed. The ectopic atrial and ventricular complexes and the episodes of pauses were excluded.

Transesophageal electrophysiologic study (TEEPS): The technique was previously described by Benson et al. ${ }^{13}$. A 6 Fr quadripolar electrode (Fiab, Esokid 4, Italy) with electrode spaced at $10 \mathrm{~mm}$ was positioned through the nares into the esophagus. The electrode was initially adjusted until the proximal electrode showed the greatest amplitude of atrial electrogram. 
Table I. Characteristics and Nodal Functions of the Patients

\begin{tabular}{lcccc}
\hline & \multicolumn{4}{c}{ Patient Groups } \\
& All & $\begin{array}{c}\text { Tilt Positive } \\
\text { (n: } 51)\end{array}$ & $\begin{array}{c}\text { Til } 33) \\
\text { (n: 18) }\end{array}$ & p value \\
\hline Age (years) & $14.01 \pm 2.79$ & $13.57 \pm 2.95$ & $14.83 \pm 2.30$ & 0.061 \\
Gender (male/female) & $21 / 30$ & $16 / 17$ & $5 / 13$ & 0.15 \\
Weight (kg) & $45.64 \pm 15.38$ & $44.39 \pm 14.28$ & $46.40 \pm 16.91$ & 0.45 \\
WP (ms) & $342.9 \pm 76$ & $378.89 \pm 57.07$ & $322.65 \pm 78.67$ & 0.011 \\
CNRT (ms) & $290 \pm 75.57$ & $292.40 \pm 73.9$ & $285.72 \pm 80.3$ & 0.76 \\
\hline
\end{tabular}

CNRT: corrected sinus node recovery time; ms: millisecond; WP: Wenckebach point

Standard 12-lead and transesophageal ECG were continuously recorded during pacing. A standard pacing protocol was applied for the evaluation of sinus and atrioventricular nodes' functions. Corrected sinus node recovery time (CNRT) and Wenckebach point (WP) values were calculated based on ECG recorded during rapid atrial pacing.

Statistical analysis: Continuous data are expressed as mean values \pm standard deviation. Univariate comparison was performed by the Student's t-test or chi-square analysis, where appropriate. A p value of $<0.05$ was considered significant.

The study protocol was approved by the institutional ethical committee.

\section{Results}

In this study, we evaluated a total of 51 children with the complaint of syncope; of these patients, 21 were males and 30 were females. HUTT was determined to be positive in 33 patients and negative in 18 patients. The major characteristics of patients depending on the results of HUTT, and their cardiac nodal functions determined by the TEPPS are indicated in Table I. Statistically significant differences did not exist between the two groups in terms of major characteristics. The cardiac nodal functions of all patients were determined to be normal. The CNRT values that show the functions of sinoatrial node were also statistically similar in two groups (p: 0.76). The WP values differed significantly between the groups (p: 0.011). Tachycardia could be induced with TEEPS in none of the patients. No complications occurred related to TEEPS.

Statistically significant differences were not observed between the groups in terms of the values of HR min, HR mean, and HR max. In the HUTT positive patient group, the values of the HF, rMSSD, and pNN50, which are the parameters of HRV, were determined to be significantly higher, compared to the HUTT negative patient group (Table II).

\section{Discussion}

The present study evaluated cardiac autonomic and cardiac nodal functions in pediatric patients suffering from vasovagal syncope. The sinus node dysfunction may be a cause of syncope due to bradycardia and decreased cerebral perfusion. However, the studies conducted on adult patients have reported that syncope was associated with neurocardiologic reflex even in patients with sinus node dysfunction ${ }^{14-16}$. Nevertheless, a value of CNRT exceeding $800 \mathrm{~ms}$ is an independent risk factor for syncope ${ }^{17}$. In our study, none of the children with vasovagal syncope had sinus node dysfunction. Moreover, the numeric value of CNRT did not differ significantly between the tilt positive and tilt negative patient groups. The sinus node dysfunction may theoretically be a cause of syncope. However, in children with syncope and normal findings on ECG and Holter monitoring, there is no need to raise concerns about sinus node dysfunction. It can be said that sinus node dysfunction is extremely rare in pediatric patients with syncope, who do not have an additional cardiac disease. When it is known that the sinus node functions are normal in children with VVS, the possible redundant concerns can also be avoided during the patient follow-up.

Brembilla-Perrot ${ }^{14}$ et al. reported that atrioventricular conduction disorder occurred in only $1 \%$ of patients with unexplained syncope 
Table II. Calculated Time-Domain and Frequency-Domain Variables in the Patients

\begin{tabular}{lccc}
\hline & $\begin{array}{c}\text { Tilt Positive Patients } \\
\text { (n: 33) }\end{array}$ & $\begin{array}{c}\text { Tilt Negative Patients } \\
\text { (n: } 18)\end{array}$ & p value \\
\hline Average heart rate $(\mathrm{bpm})$ & $78.09 \pm 15.8$ & $83.8 \pm 9.87$ & 0.169 \\
Minimum heart rate $(\mathrm{bpm})$ & $49.0 \pm 7.04$ & $48.77 \pm 6.7$ & 0.9 \\
Maximum heart rate $(\mathrm{bpm})$ & $156.15 \pm 17.1$ & $162.27 \pm 14.5$ & 0.2 \\
SDNN (ms) & $151.24 \pm 45.6$ & $151.88 \pm 42.9$ & 0.96 \\
SDNNi (ms) & $70.81 \pm 21.8$ & $60.16 \pm 18.78$ & 0.08 \\
SDANN (ms) & $127.69 \pm 36.2$ & $138.88 \pm 41$ & 0.3 \\
rMSSD (ms) & $49.93 \pm 22.67$ & $37.55 \pm 11.7$ & $0.03^{*}$ \\
pNN50 (\%) & $20.81 \pm 11.09$ & $13.88 \pm 8$ & $0.02^{*}$ \\
Total Power (ms $\left.{ }^{2}\right)$ & $7,031.53 \pm 8,804.13$ & $3,605.38 \pm 2,084.14$ & 0.11 \\
VLF (ms & $3,520.36 \pm 2,447.92$ & $2,367.72 \pm 1,551.81$ & 0.06 \\
LF $\left(\mathrm{ms}^{2}\right)$ & $799.92 \pm 423.3$ & $1,053.46 \pm 525.44$ & 0.08 \\
HF $\left(\mathrm{ms}^{2}\right)$ & $629.54 \pm 332.37$ & $403.96 \pm 199.3$ & $0.01^{*}$ \\
\hline
\end{tabular}

bpm: beats per minute; LF: low-frequency component; HF: high-frequency component; ms: millisecond; pNN50: percentage of successive normal sinus R-R intervals longer than $50 \mathrm{~ms}$; rMSSD: root mean square of the successive normal sinus R-R interval difference; SDNN: standard deviation of all normal sinus R-R intervals during 24 h; SDNNi: standard deviation of all normal sinus R-R intervals for all 5-min segments; SDANN: the standard deviation of the average normal sinus R-R intervals for all 5-min segments, TEEPS: transesophageal electrophysiological study; VLF: very-low-frequency component

and a normal ECG. In our study, no patient had AV conduction disorder. However, the WP value in the patient group with a positive tilt test was significantly higher compared to the patient group with a negative tilt test. We consider that this is associated with decreased dromotropy developing due to an increased parasympathetic tonus in the patient group with a positive tilt test.

It was reported that undiagnosed supraventricular tachycardia might be a cause of syncope in a study evaluating the diagnostic efficacy of transesophageal electrophysiologic study in 154 patients with unexplained syncope and without an additional cardiac disease. Brembilla-Perrot et al. ${ }^{11}$ included in this study the patients without a documented arrhythmia and with a normal ECG, but suffering from palpitation. Moreover, the ages of patients in this study ranged between 16 years and 87 years. In our study, despite the stimulation of atrium with TEEPS, we could induce supraventricular tachycardia in none of these paediatric patients. Our cases did not report a complaint of palpitation, and this fact may explain the controversial results.

When the HRV parameters were investigated in the study groups, the HRV parameters that indicate the parasympathetic tonus differed between the tilt positive and tilt negative patient groups. HF $\left(\mathrm{ms}^{2}\right)$, rMSSD (ms), and pNN50 (\%) are the HRV parameters that show parasympathetic activity (5). The difference in the values of HF $\left(\mathrm{ms}^{2}\right), \mathrm{rMSSD}$ (ms), and pNN50 (\%) in the tilt positive patients is a finding, which is in favor of increased parasympathetic activity occurring in patients with vasovagal syncope. This finding is consistent with the determination of differences in TEEPS and WP between the two groups. The difference in autonomic tonus between the tilt positive and tilt negative patient groups has been shown by HRV parameters and also by the AV conduction characteristics.

In pediatric patients suffering from VVS, increased parasympathetic tonus predominates in the autonomic nervous system. This increased activity in parasympathetic system can be shown by evaluating the HRV parameters. Significant impairment in cardiac nodal functions should not be expected in children suffering from VVS. However, the WP, which is the indicator of atrioventricular node function, may be prolonged in cases with VVS. The change in WP in these patients may be due to increased parasympathetic tonus occurring 
in these cases.

\section{REFERENCES}

1. Kapoor WN. Syncope. N Engl J Med 2000; 343:18561862.

2. McHarg ML, Shinnar S, Rascoff H, Walsh CA. Syncope in childhood. Pediatr Cardiol, 1999; 18: 367-371.

3. Moya A, Sutton R, Ammirati F, et al. Guidelines for the diagnosis and management of syncope (version 2009). Eur Heart J 2009; 30: 2631-2671.

4. Alehan D, Ayabakan C, Ozer S. Heart rate variability and autonomic nervous system changes in children with vasovagal syncope. Pacing Clin Electrophysiol 2002; 25: 1331-1338.

5. Task Force of the European Society of Cardiology and the North American Society of Pacing and Electrophysiology. Heart rate variability. Standards of measurement, physiological interpretation, and clinical use. Circulation 1996; 93: 1043-1065.

6. Khalil M, Hessling G, Bauch M, Maier C, Dickhaus $\mathrm{H}$, Ulmer HE. Sympathovagal imbalance in pediatric patients with neurocardiogenic syncope during asymptomatic time periods. J Electrocardiol 2004; 37(Suppl): 166-170.

7. Shim SH, Park SY, Moon SN, et al. Baseline heart rate variability in children and adolescents with vasovagal syncope. Korean J Pediatr 2014; 57: 193-198.

8. Longin E, Reinhard J, von Buch C, Gerstner T, Lenz T, König S. Autonomic function in children and adolescents with neurocardiogenic syncope. Pediatr Cardiol 2008; 29: 763-770.

9. Sehra R, Hubbard JE, Straka SP, et al. Autonomic changes and heart rate variability in children with neurocardiac syncope. Pediatr Cardiol 1999; 20: 242-
247.

10. Stewart JM, Erb M, Sorbera C. Heart rate variability and the outcome of head-up tilt in syncopal children. Pediatr Res 1996; 40: 702-709.

11. Brembilla-Perrot B, Beurrier D, Houriez P, et al. Utility of transesophageal atrial pacing in the diagnostic evaluation of patients with unexplained syncope associated or not with palpitations. Int J Cardiol 2004; 96: 347-353.

12. Erdoğan I, Ozer S, Karagöz T, Sahin M, Celiker A. Clinical importance of transesophageal electrophysiologic study in the management of supraventricular tachycardia in children. Turk J Pediatr 2009; 51: 578-581.

13. Benson DW, Dunnigam A, Benditt DG, Pritzker ME, Thomson TR. Transesophageal study of in infant supraventricular tachycardia. Electrophysiologic characteristics. Am J Cardiol 1983; 52: 1002-1006.

14. Brembilla-Perrot B, Claudon O, Beurrier D, et al. Syncope in patients with normal ECG and without cardiac disease: value of ambulatory esophageal electrophysiology in determining etiology. Arch Mal Coeur Vaiss 2002; 95: 883-889.

15. Oddone D, Brignole M, Menozzi C, Gianfranchi L, Lolli G. Spontaneous occurrence of the induced cardioinhibitory vasovagal reflex. Pacing Clin Electrophysiol 1991; 14: 415-419.

16. Brignole $\mathrm{M}$, Menozzi C, Bottoni N, et al. Mechanisms of syncope caused by transient bradycardia and the diagnostic value of electrophysiologic testing and cardiovascular reflexivity maneuvers. Am J Cardiol 1995; 76: 273-278.

17. Alboni P, Menozzi C, Brignole M, et al. Effects of permanent pacemaker and oral theophylline in sick sinus syndrome the THEOPACE study: A randomized controlled trial. Circulation 1997; 96: 260-266. 